\title{
Precision calculations for extended Higgs sectors with and without supersymmetry
}

\section{Wolfgang Hollik*}

Max Planck Institut für Physik, 80805 Munich

E-mail: hollik@mpp.mpg.de

Calculations of electroweak precision observables is reviewed for extensions of the Standard Model with two Higgs doublets in the symmetry breaking sector. Results are given for supersymmetric and non-supersymmetric versions of two-doublet models including recent constraints from the experiments ath the LHC.

Loops and Legs in Quantum Field Theory

24-29 April 2016

Leipzig, Germany

* Speaker. 


\section{Introduction}

High-precision experiments at electron-positron and hadron colliders together with highly accurate measurements at low energies have imposed stringent tests on the Standard Model (SM) and possible extensions. The experimental accuracy in the electroweak observables is sensitive to the quantum effects and requires the highest standards on the theoretical side as well. A sizeable amount of theoretical work has contributed over more than two decades to a steadily rising improvement of the SM predictions and also for specific new physics scenarios like supersymmetric extensions. The highly accurate measurements and theoretical predictions, at the level of $0.1 \%$ precision and better, provide unique tests of the quantum structure of the SM having been impressively confirmed by the discovery of a Higgs particle [1]. Moreover, it opens the possiblity to obtain indirect informations on potential other heavy new physics beyond the SM, in particular on the not yet sufficiently explored scalar sector.

\section{Electroweak Precision Observables and the Standard Model}

The possibility of performing precision tests is based on the formulation of the Standard Model as a renormalizable quantum field theory preserving its predictive power beyond tree-level calculations.

Vector boson masses. A particularly important role is given by the correlation of the vectorboson masses $M_{W}$ and $M_{Z}$ in terms of the Fermi constant $G_{F}$. Calculating the effective Fermi constant via the muon lifetime within the Standard Model yields the relation

$$
M_{W}^{2}\left(1-\frac{M_{W}^{2}}{M_{Z}^{2}}\right)=\frac{\pi \alpha}{\sqrt{2} G_{F}}(1+\Delta r)
$$

where the higher-order contributions are summarized in the quantity $\Delta r$. The one-loop result [2] has been improved over the last two decades by complete QCD [3] and electroweak [4] two-loop terms as well as the leading 3- and 4-loop contributions [5], establishing thus a powerful relation that can be used to predict $M_{W}$ within the SM (or possible extensions), to be confronted with the experimental result for $M_{W}$. The quantity $\Delta r$ depends on the entire set of input parameters. A basic universal entry is the QED-shift $\Delta \alpha$ of the electromagnetic fine structure constant resulting from the light-fermion part of the photon vacuum polarization. $\Delta \alpha$ and the quantum correction to the $\rho$-parameter, given through the $W, Z$ self-energies,

$$
\Delta \rho=\frac{\Sigma_{Z}(0)}{M_{Z}^{2}}-\frac{\Sigma_{W}(0)}{M_{W}^{2}},
$$

constitute the dominating and universal terms of $\Delta r$, at one-loop order given by

$$
\Delta r=\Delta \alpha+\frac{c_{W}^{2}}{s_{W}^{2}} \Delta \rho+\cdots, \quad c_{W}=\cos \theta_{W}=\frac{M_{W}}{M_{Z}}
$$

with

$$
\Delta \rho=3 \frac{G_{F} m_{t}^{2}}{8 \pi^{2} \sqrt{2}}+\cdots \simeq 0.0094
$$


$Z$ boson observables. With $M_{Z}$ used as a precise input parameter, together with $\alpha$ and $G_{F}$, the predictions for the $Z$ width, partial widths and asymmetries can conveniently be calculated in terms of effective neutral current coupling constants for the various fermions in the weak current

$$
J_{v}^{\mathrm{NC}}=\left(\sqrt{2} G_{F} M_{Z}^{2}\right)^{1 / 2}\left(g_{V}^{f} \gamma_{v}-g_{A}^{f} \gamma_{v} \gamma_{5}\right)
$$

from which (fermion-specific) effective weak mixing angles $\sin _{\text {eff }}^{f}$ can be derived:

$$
g_{V}^{f} / g_{A}^{f}=1-2 Q_{f} \sin ^{2} \theta_{\mathrm{eff}}^{f} .
$$

For the leptonic mixing angle, the complete electroweak fermionic and bosonic two-loop contributions have become available [6], together with the universal terms of higher order mentioned already in the context of $\Delta r$, especially the higher-order contributions to the $\rho$-parameter (2.2), yielding

$$
\sin ^{2} \theta_{\text {eff }}^{f}=s_{W}^{2}+c_{W}^{2} \Delta \rho+\cdots .
$$

The predictions for the various widths include the fermionic contributions at the electroweak twoloop level [7], i.e. those with at least one closed fermion loop.

With the meanwhile very precisely measured Higgs-boson mass [8] of $M_{H}=125 \pm 0.24 \mathrm{GeV}$ the SM input is now completely determined and the SM predictions for the set of precision observables are uniqe, being in overall good agreement with the data. This improves the sensitivity to physics beyond the SM and makes constraints on parameters of extended models quite severe.

\section{Two Higgs doublets within the MSSM}

The Higgs sector. Among the extensions of the Standard Model, the minimal supersymmetric standard model (MSSM) is the theoretically favoured scenario as the most predictive framework beyond the Standard Model. A light Higgs boson, as empirically observed, would find a natural explanation by the structure of the Higgs potential

The MSSM accommodates two isospin doublets of Higgs fields in the symmetry breaking sector. The corresponding five physical Higgs particles consist of two $C P$-even neutral bosons $h^{0}, H^{0}$, a $C P$-odd $A^{0}$ boson, and a pair of charged Higgs particles $H^{ \pm}$. Since the quartic couplings of the Higgs potential are combinations of the gauge couplings and thus not free quantitities, the tree-level Higgs potential has only two independent parameters. Hence, the tree-level masses of the Higgs particles can all be expressed in terms of the $A^{0}$ boson mass, $M_{A}$, and the ratio of the two vacuum expectation values, $v_{2} / v_{1}=\tan \beta$ :

$$
\begin{aligned}
& m_{H^{ \pm}}^{2}=M_{A}^{2}+M_{W}^{2}, \\
& m_{H, h}^{2}=\frac{1}{2}\left(M_{A}^{2}+M_{Z}^{2} \pm \sqrt{\left(M_{A}^{2}+M_{Z}^{2}\right)^{2}-4 M_{A}^{2} M_{Z}^{2} \cos ^{2} 2 \beta}\right) .
\end{aligned}
$$

These relations are, however, strongly influenced by loop contributions, in particular the mass of the lightest Higgs boson $h^{0}$. For large $M_{A}$, the $h^{0}$ particle behaves like the Standard Model Higgs boson, but its mass is dependent on basically all the parameters of the model. A definite prediction of the MSSM is the existence of a light Higgs boson, and the mass itself is an additonal 
precision observable with sensitivity to the supersymmetric mass parameters. A typical example of a spectrum is shown in Figure 1.

Present spectrum calculations comprise the complete one-loop terms and the leading terms at two-loop order originating from the strong interaction and the $t, b$ Yukawa couplings. They are summarized and incorporated, for example, in the public code FEYNHiggs [9].

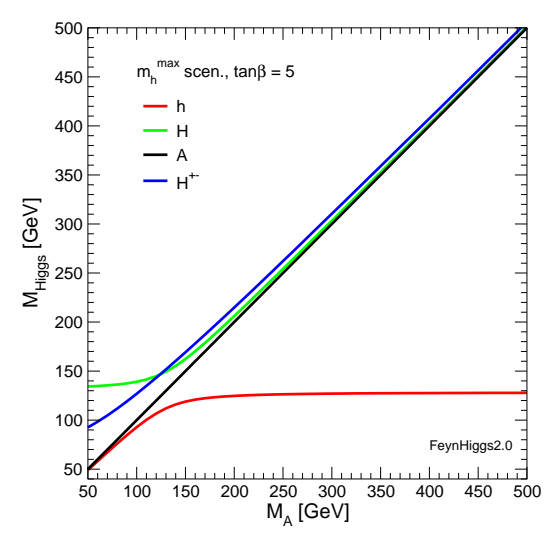

Figure 1: Example of the Higgs boson mass spectrum in the MSSM.

Precision observables. The structure of the MSSM as a renormalizable quantum field theory allows a similarly complete calculation of the electroweak precision observables as in the Standard Model in terms of one Higgs mass (usually taken as $M_{A}$ ) and $\tan \beta$, together with the set of SUSY soft-breaking parameters fixing the chargino/neutralino and scalar fermion sectors. Early one-loop calculations were done in refs. [11, 12, 13, 14]. The general discussion of renormalization of the MSSM to all orders with implications on the structure of the counter terms is given in [10]. Recent calculations for $\Delta r$ [16] and for the $Z$ boson observables [17] are complete at the one-loop level and moreover incorporate all available Standard Model two-loop and higher-order terms as well as the leading non-standard two-loop contributions through the $\rho$-parameter $[15,18]$ arising from the strong interaction and the Yukawa couplings. A review on precision observables in the MSSM can be found in [19]. Formally equivalent to the Standard Model, the relation

$$
\frac{G_{F}}{\sqrt{2}}=\frac{\pi \alpha}{M_{W}^{2}\left(1-M_{W}^{2} / M_{Z}^{2}\right)} \cdot\left[1+\Delta r\left(m_{t}, X\right)\right]
$$

determines the $W$ mass $M_{W}=M_{W}\left(\alpha, G_{F}, M_{Z}, m_{t}, X\right)$ in terms of the non-standard parameters $X$ from the Higgs and SUSY-particle sectors under the constraint that either $m_{h}$ or $m_{H}$ is given by the experimental value of the Higgs-boson mass. An evaluation on the basis of all available higherorder terms from the Standard Model and the MSSM in the calculation and with the implications of LHC results is given in [20]. The masses and mixings of the Higgs bosons in the loop diagrams for $\Delta r$ are taken from FEYNHIGGS including higher order contributions.

As a typical example, Figure 2 displays the range of predictions for $M_{W}$ in the Standard Model and in the MSSM [20], together with the present experimental errors on $m_{t}$ and $M_{W}$. The MSSM range is based on the assumption that the $C P$-even $h^{0}$ is the observed Higgs particle at $125 \mathrm{GeV}$ (the given error in the figure incorporates also the theoretical uncertainty in the prediction of the $h^{0}$ mass $M_{h}$ from the model parameters) and is moreover compatible with the experimental constraints 


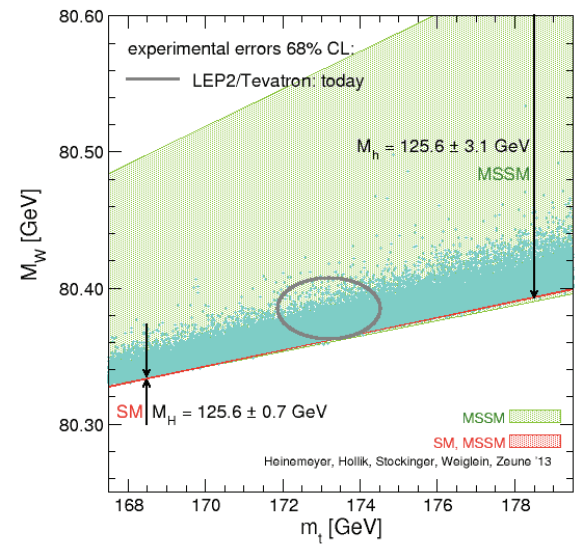

Figure 2: The $W$ mass in the SM (red line) and MSSM (green area) respecting bounds from the Higgs mass and on SUSY particles.
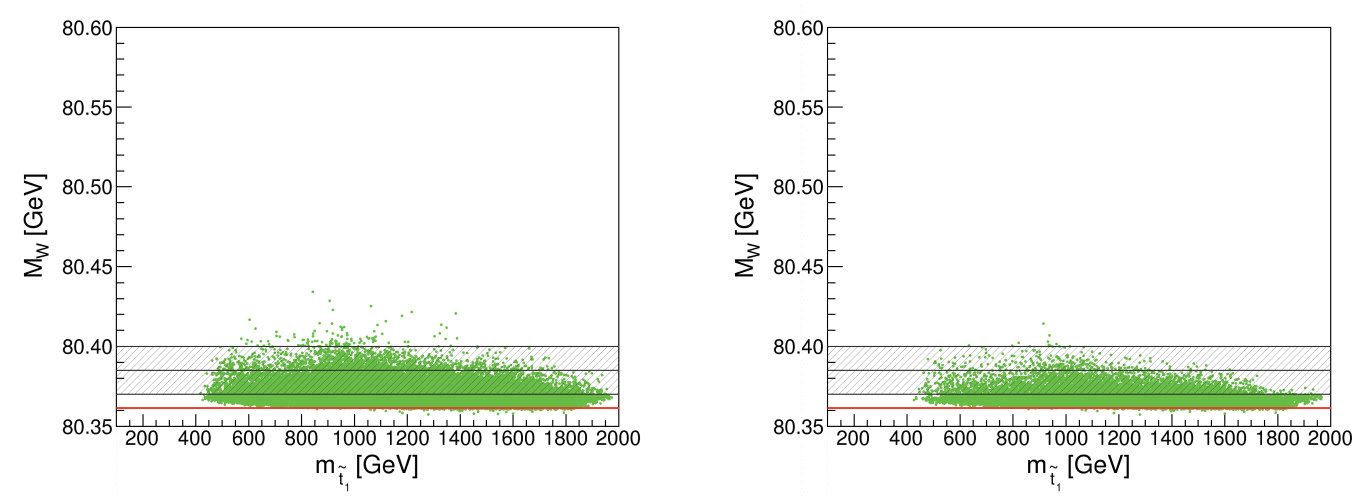

Figure 3: The $W$ mass in the SM (red line) and in the MSSM with $m_{\tilde{q}}, m_{\tilde{g}}>1200 \mathrm{GeV}, \mathrm{m}_{\tilde{b}}>1000 \mathrm{GeV}$. Right: additional constraints on charginos and sleptons with masses $>500 \mathrm{GeV}$.

from Higgs boson searches. The dark area indicates the range where the top- and bottom-squarks are heavier than $500 \mathrm{GeV}$ and squarks of the 1 st and 2 nd generation and the gluino are heavier than $1200 \mathrm{GeV}$. Constraining also $\tilde{b}$-squarks to be heavier than $1 \mathrm{TeV}$ [20], one obtains the green regions in Figure 3 in dependence of the lighter stop mass $m_{\tilde{t}_{1}}$, overlapping nicely with the $\pm 1 \sigma$-range of the $W$ mass, even with rather heavy electroweak SUSY particles as displayed in the right plot of Figure 3.

\section{General Two-Higgs-Doublet Model}

The Higgs sector. In the CP-conserving Two-Higgs-Doublet Model (THDM) the Higgs potential involves the two scalar doublets, with vacua $v_{1}$ and $v_{2}$, as a 4th-order polynomial with quadratic and quartic coefficients $\lambda_{1}, \ldots \lambda_{6}$, which differently from the supersymmetric case are independent parameters:

$$
V\left(\Phi_{1}, \Phi_{2}\right)=
$$




$$
\begin{aligned}
& \lambda_{1}\left(\Phi_{1}^{+} \Phi_{1}-v_{1}^{2}\right)^{2}+\lambda_{2}\left(\Phi_{2}^{+} \Phi_{2}-v_{2}^{2}\right)^{2}+\lambda_{3}\left[\left(\Phi_{1}^{+} \Phi_{1}-v_{1}^{2}\right)+\left(\Phi_{2}^{+} \Phi_{2}-v_{2}^{2}\right)\right]^{2}+ \\
& \lambda_{4}\left[\left(\Phi_{1}^{+} \Phi_{1}\right)\left(\Phi_{2}^{+} \Phi_{2}\right)-\left(\Phi_{1}^{+} \Phi_{2}\right)\left(\Phi_{2}^{+} \Phi_{1}\right)\right]+\lambda_{5}\left[\operatorname{Re}\left(\Phi_{1}^{+} \Phi_{2}\right)-v_{1} v_{2}\right]^{2}+\lambda_{6}\left[\operatorname{Im}\left(\Phi_{1}^{+} \Phi_{2}\right)\right]^{2} .
\end{aligned}
$$

The mass eigenstates comprise, similar to the MSSM, two $C P$-even neutral scalars $h^{0}, H^{0}$, one neutral $C P$-odd "pseudoscalar" $A^{0}$, and a pair of charged Higgs bosons $H^{ \pm}$. The corresponding fields, together with the three Goldstone fields $G^{0}, G^{ \pm}$, are obtained from diagonalizing the quadratic part of the potential (4.1). Formally in the same way as in the MSSM, the angle $\beta$ with $\tan \beta=v_{2} / v_{1}$, yields $A_{0}$ and $H^{ \pm}$, and another angle $\alpha$ leads to $h^{0}$ and $H^{0}$; however, now the two angles and all masses $m_{h}, m_{H}, m_{A}, m_{H^{ \pm}}$are free and independent parameters. The $\lambda$-parameters of the potential can then be re-expressed in terms of the masses and mixing angles in the following way,

$$
\begin{aligned}
& \lambda_{1}=\frac{1}{4 v^{2} \cos ^{2} \beta}\left[m_{H}^{2}+m_{h}^{2}+\left(m_{H}^{2}-m_{h}^{2}\right) \frac{\cos (2 \alpha+\beta)}{\cos \beta}\right]+\lambda_{3}\left(-1+\tan ^{2} \beta\right), \\
& \lambda_{2}=\frac{1}{4 v^{2} \sin ^{2} \beta}\left[m_{H}^{2}+m_{h}^{2}+\left(m_{h}^{2}-m_{H}^{2}\right) \frac{\sin (2 \alpha+\beta)}{\sin \beta}\right]+\lambda_{3}\left(-1+\cot ^{2} \beta\right), \\
& \lambda_{4}=\frac{2 m_{H^{ \pm}}^{2}}{v^{2}}, \quad \lambda_{5}=\frac{2}{v^{2}} \frac{\sin 2 \alpha}{\sin 2 \beta}\left(m_{H}^{2}-m_{h}^{2}\right)-4 \lambda_{3}, \quad \lambda_{6}=\frac{2 m_{A}^{2}}{v^{2}},
\end{aligned}
$$

where $v^{2}=v_{1}^{2}+v_{2}^{2}$. Since (4.1) contains 8 parameters, with one constraint on $v$ from the gauge sector, one of the $\lambda$-coefficients is still unconstrained and has to be treated as another free parameter in addition to the set of masses and mixing angles. By convention, the quantity $\lambda_{5}$ is usually chosen. For $\alpha=\frac{\pi}{2}-\beta$, the alignment limit, the $h^{0}$ particle behaves like the Standard Model Higgs boson and can be identified with the observed scalar boson at $125 \mathrm{GeV}$.

Precision observables. Also for the general THDM, the calculation of electroweak precision observables has a long history [21, 22, 23, 24, 25]. In a recent study [26] they are evaluated on the basis of all available higher-order terms from the Standard Model together with the complete oneloop non-standard terms and the leading non-standard two-loop contributions from the top-Yukawa coupling and the Higgs self-couplings through the $\rho$-parameter (2.2) in the approximation of the gaugeless limit. Figure 4 shows the sensitivity to mass splittings between the neutral and charged Higgs particles, which is essentially an effect of $\Delta \rho$. At the two-loop level, $\Delta \rho$ becomes dependent in addition on the self-coupling $\lambda_{5}$ and on $\tan \beta$, as displayed in Figure 5.
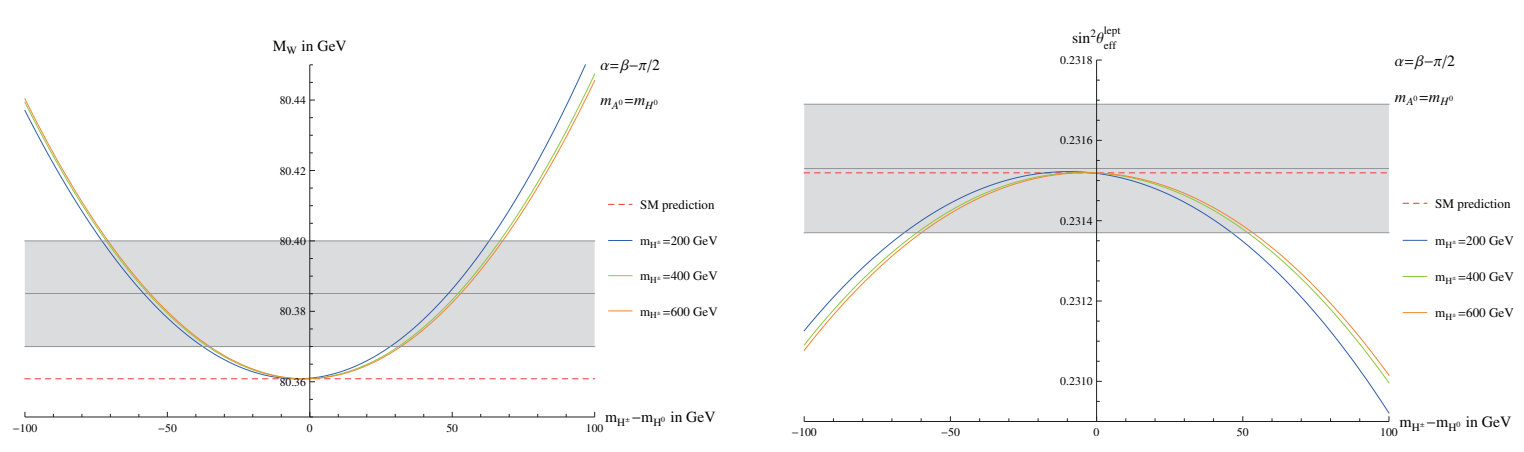

Figure 4: The $W$ mass and the effective mixing angle in the THDM. The shaded area indicates the experimental $\pm 1 \sigma$-range, and the red line is the Standard Model prediction. 


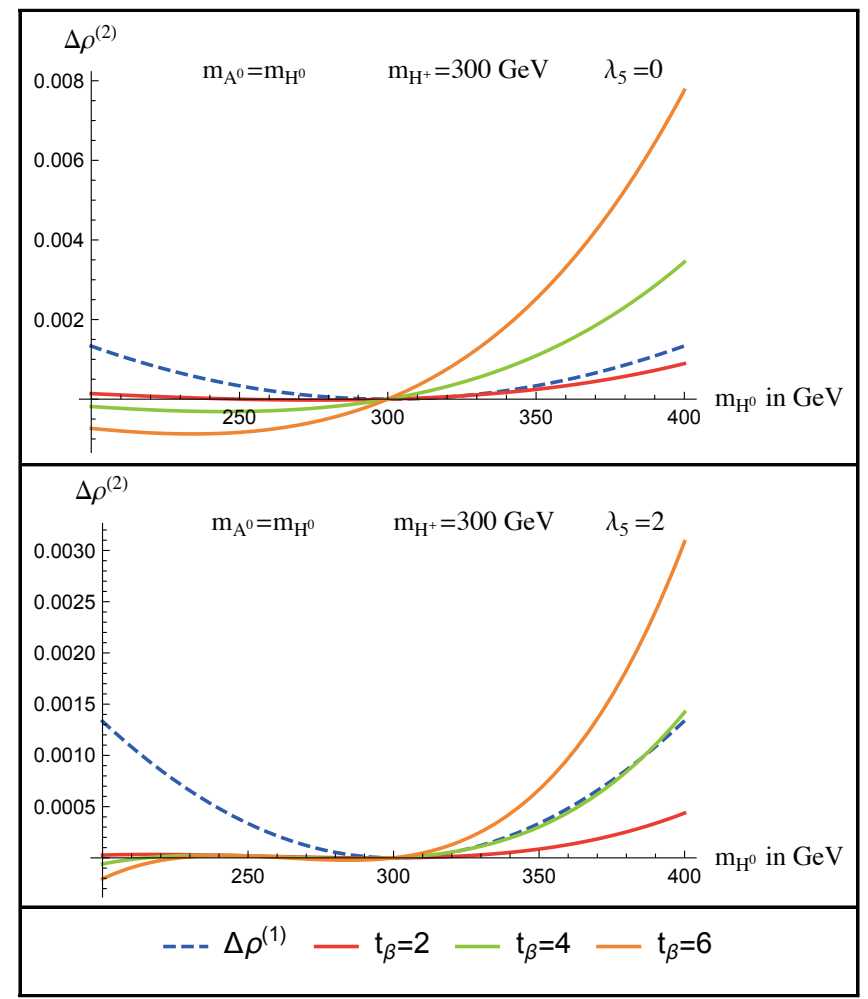

Figure 5: Two-loop contributions to the $\rho$-parameter from the non-standard scalars in the alignment limit. The blue dashed line is the on-loop result.

\section{References}

[1] G. Aad et al. [ATLAS Collaboration], Phys. Lett. B 716 (2012) 1; S. Chatrchyan et al. [CMS Collaboration], Phys. Lett. B 716 (2012) 30

[2] A. Sirlin, Phys. Rev. D 22, 971 (1980); W.J. Marciano, A. Sirlin, Phys. Rev. D 22, 2695 (1980)

[3] B.A. Kniehl, Nucl. Phys. B 347 (1990) 89; F. Halzen, B.A. Kniehl, Nucl. Phys. B 353 (1991) 567; B.A. Kniehl, A. Sirlin, Nucl. Phys. B 371 (1992) 141, Phys. Rev. D 47 (1993) 883; A. Djouadi, P. Gambino, Phys. Rev. D 49 (1994) 3499

[4] A. Freitas, W. Hollik, W. Walter, G. Weiglein, Phys. Lett. B 495 (2000) 338, Nucl. Phys. B 632 (2002) 189; M. Awramik, M. Czakon, Phys. Rev. Lett. 89 (2002) 241801; Phys. Lett. B 568, 48 (2003); A. Onishchenko, O. Veretin, Phys. Lett. B 551 (2003) 111; M. Awramik, M. Czakon, A. Onishchenko, O. Veretin, Phys. Rev. D 68 (2003) 053004; G. Degrassi, P. Gambino, P. P. Giardino, JHEP 1505 (2015) 154

[5] J. van der Bij, K. Chetyrkin, M. Faisst, G. Jikia, T. Seidensticker, Phys. Lett. B 498 (2001) 156; M. Faisst, J. Kühn, T. Seidensticker, O. Veretin, Nucl. Phys. B 665 (2003) 649; Y. Schroder, M. Steinhauser, Phys. Lett. B 622 (2005) 124; K. Chetyrkin, M. Faisst, J. Kühn, P. Maierhofer, Phys. Rev. Lett. 97 (2006) 102003; R. Boughezal, M. Czakon, Nucl. Phys. B 755 (2006) 221; R. Boughezal, J.B. Tausk, J.J. van der Bij, Nucl. Phys. B 725 (2005) 3; 713 (2005) 278 
[6] M. Awramik, M. Czakon, A. Freitas, G. Weiglein, Phys. Rev. Lett. 93 (2004) 201805; M. Awramik, M. Czakon, A. Freitas, Phys. Lett. B 642 (2006) 563, JHEP 0611, 048 (2006); W. Hollik, U. Meier, S. Uccirati, Nucl. Phys. B 731 (2005) 213, B 765 (2007), 154 (2007); Phys. Lett. B 632, 680 (2006)

[7] A. Freitas, JHEP 1404 (2014) 070

[8] G. Aad et al. [ATLAS and CMS Collaborations], Phys. Rev. Lett. 114 (2015) 191803

[9] G. Degrassi, S. Heinemeyer, W. Hollik, P. Slavich, G. Weiglein, Eur. Phys. J. C 28 (2003) 133; M. Frank, T. Hahn, S. Heinemeyer, W. Hollik, H. Rzehak, G. Weiglein, JHEP 0702 (2007) 047; S. Heinemeyer, W. Hollik, G. Weiglein, Comput. Phys. Commun. 124 (2000) 76; T. Hahn, S. Heinemeyer, W. Hollik, H. Rzehak, G. Weiglein, Comput. Phys. Commun. 180 (2009) 1426, Nucl. Phys. Proc. Suppl. 205-206 (2010) 152

[10] W. Hollik, E. Kraus, M. Roth, C. Rupp, K. Sibold, D. Stöckinger, Nucl. Phys. B 639 (2002) 3

[11] J. A. Grifols, J. Sola, Phys. Lett. B 137 (1984) 257; J. A. Grifols, J. Sola, Nucl. Phys. B 253 (1985) 47

[12] P. Chankowski, A. Dabelstein, W. Hollik, W. Mosle, S. Pokorski, J. Rosiek, Nucl. Phys. B 417 (1994) 101.

[13] D. Garcia, J. Sola, Mod. Phys. Lett. A 9 (1994) 211

[14] D. Pierce, J. Bagger, K. Matchev, R. Zhang, Nucl. Phys. B 491 (1997) 3

[15] J. Haestier, S. Heinemeyer, D. Stöckinger, G. Weiglein, JHEP 0512 (2005) 027

[16] Heinemeyer, W. Hollik, D. Stöckinger, A.M. Weber, G. Weiglein, JHEP 0608, 052 (2006)

[17] Heinemeyer, W. Hollik, A.M. Weber, G. Weiglein, JHEP 0804, 039 (2008)

[18] A. Djouadi, P. Gambino, S. Heinemeyer, W. Hollik, C. Jünger and G. Weiglein, Phys. Rev. Lett. 78, 3626 (1997); Phys. Rev. D 57, 4179 (1998); S. Heinemeyer, G. Weiglein, JHEP 0210, 072 (2002);

[19] S. Heinemeyer, W. Hollik, G. Weiglein, Phys. Rept. 425, 265 (2006)

[20] S. Heinemeyer, W. Hollik, G. Weiglein, L. Zeune, JHEP 1312 (2013) 084

[21] S. Bertolini, Nucl. Phys. B 272 (1986) 77

[22] W. Hollik, Z. Phys. C 32 (1986) 291, Z. Phys. C 37 (1988) 569

[23] A. Denner, R. J. Guth, W. Hollik, J. H. Kühn, Z. Phys. C 51 (1991) 695

[24] P. H. Chankowski, M. Krawczyk, J. Zochowski, Eur. Phys. J. C 11 (1999) 661

[25] D. Lopez-Val, J. Sola, Eur. Phys. J. C 73 (2013) 2393

[26] S. Hessenberger, Two Higgs Doublet Models and electroweak precision observables, Diploma Thesis, Technische Universität München 2013; S. Hessenberger, W. Hollik, to appear 